\title{
Video reviews of clinical encounters: can authentic feedback improve third-year student physicians' interpersonal skills?
}

\author{
Heather McCarthy ${ }^{1 *}$ and Janet Lynn Roseman ${ }^{2}$ \\ ${ }^{1}$ Medical Education \& Osteopathic Principles and Practice, Nova Southeastern University, USA \\ ${ }^{2}$ Integrative Medicine, Nova Southeastern College of Osteopathic Medicine, USA
}

\begin{abstract}
Objective: We have conducted this study to examine the efficacy of video reviews for third year osteopathic medical students after interactions with standardized patients in an effort to improve interpersonal skills.

Methods: We conducted group video feedback sessions with 392 osteopathic medical students who attended M3 Come Home Day, an innovative program for third year osteopathic medical students who return to Nova Southeastern College of Osteopathic Medicine's Ft. Lauderdale campus during their third year of medical school training. The 392 medical students completed a voluntary survey at the end of the group feedback session to help evaluate the efficiency of their participation in the all-day program. Although, 9 questions were asked in the anonymous survey, this paper will concentrate on 3 of the 9 questions asked. An IRB was filed and approved.
\end{abstract}

Results: The results indicated that during the identified years (2011-2012,2012-2013) participants overwhelmingly felt that the feedback portion of the M3 Come Home Day Curriculum was not only a positive experience for students, but indicated that this program was valuable for future participants.

Conclusions: Our study has found that the participants in this study overwhelmingly indicated that the provision of video review feedback sessions was very helpful to them and it is hoped that in the future we can examine how to improve this innovative program so that the osteopathic medical students can improve their interpersonal skills.

\section{Introduction}

The use of patient simulation encounters is not a new teaching method for medical students and according to Westwood, (2013) "one third of all medical schools use patient simulation techniques" to improve medical students' skills for training in patient/physician encounters. Although, the use of patient simulation is not a new technique, the M3 Come Home Day program at Nova Southeastern College of Osteopathic Medicine is an innovative model for embracing that training. Fundamental in the practice of medicine is the need for physicians (student or professional) to develop and improve their interpersonal skills with patients. Osteopathic medical students in particular are usually called to the osteopathic profession because of its emphasis on "the whole person". According to Peterson, Porter and Calhoun, "The use of standardized patients or actors simulating patient encounters has proved both effective in nursing, dental and medical education."b However, there is little literature that identifies the efficacy of simulated patient encounters with third year osteopathic medical students.

In an effort to provide innovative curriculum to improve osteopathic student physicians' interpersonal and professional acumen,

${ }^{\mathrm{a}}$ Westwood, J.D., "Medicine Meets Virtual Reality: Accelerating Change in Health Care.", pg.25-28.IOS Press, 2002.

b (Sept. 2012; 4(3): 351-356. Eleanor B. Peterson, MD, Melissa B. Porter, MD, and Aaron W. Calhoun, MD: A Simulation-Based Curriculum to Address Relational Crises in Medicine, Journal of Graduate Medical Education, Sep 2012: 4 (3): 351-356.
Nova Southeastern College of Osteopathic Medicine established an innovative educational initiative in 2009 entitled: "M3 Come Home Day." The objective for this program was to provide creative medical education for third year osteopathic medical students to help improve their patient encounters in the area of interpersonal skills. We hoped that through their video reviews with simulated patients that they would obtain an increased consciousness of the importance of interpersonal communications with patients. During the M3 Come Home Day Program, third year osteopathic medical students on rotation at sites across the country return to campus twice a year in order to reconnect with the medical school, receive career guidance, and improve clinical skills, osteopathic manipulation techniques and interpersonal skills. Day One of the two-day curriculum includes career guidance from experienced professionals, and separately they further enhance their osteopathic manipulative skills while working with faculty and patients in the clinics on campus. On Day Two, medical students experience

${ }^{\star}$ Correspondence to: Heather McCarthy, Assistant Professor, Educational Counseling, Medical Education and OPP, USA, Tel: (954) 262-1494; E-mail: heatherc@nova.edu

Key words: interpersonal skills, video reviews, feedback, student physicians, osteopathic medicine

Received: June 07, 2018; Accepted: June 13, 2018; Published: June 16, 2018 
mannequin simulation as well as standardized patient encounters with feedback provided by two faculty members. During 2011-2013, 392 osteopathic medical students have completed a one-page survey regarding their participation in the mannequin simulation and the standardized patient encounters. The authors chose to concentrate on the feedback aspect that the osteopathic medical students received. The student physicians met in the simulation lab and had the opportunity to practice their clinical and interpersonal skills with standardized/ simulated patients.

After the osteopathic student physician's experiences in the simulation lab with standardized patients, students met in small groups to review their recorded clinical encounters and receive feedback from their peers and two faculty members. M3 Come Home Day is required and the students who attend volunteer to watch their videos during the two-hour session. The ability to re-examine one's clinical and interpersonal skills, although awkward at first, has shown to be a powerful programmatic medical educational tool and based on our research, the M3 Come Home Day program has shown to be quite valuable.

This paper will examine in depth the data received from a short survey that was implemented. The data suggests and supports that the M3 Come Home Day program was a positive experience for the participants and provided osteopathic student physicians' important feedback for both their interpersonal and professional skills during a simulated patient encounter.

\section{Methods}

The study was anonymous and provided opportunities for medical students to access the M3 Come Home program by completion of a survey.

Participants and Sample Size: 392 students completed the voluntary survey at the end of the group feedback session without identified names for each questionnaire. The 9 questions inquiry provided data to help evaluate the efficacy of each session. Each student physician attended both the mannequin simulation and the patient simulation experience during the program. The questions were created to help measure how student physicians responded to the M3 Come Home Day Program. For the purposes of this paper, we will concentrate on 3 of the 9 questions that concerned participant's opinions on the efficacy of the feedback sessions during the M3 Come Home Day Program.

Data Collection: During the feedback sessions, several medical students volunteered to review their video of their simulated patient encounter held earlier that day. The assembled group watched the videos, provided feedback to one another, and received feedback from the faculty mentors for the program. The survey was then distributed. Participants were able to select from a range of responses including:

\footnotetext{
${ }^{c}$ The definition of standardized/simulated patients according to the Association for Standardized Patient Educators is as follows: these patients are individuals who are trained to portray a patient with a specific condition in a realistic, standardized and repeatable way. Standardized/simulated patients can be used for teaching and assessment of learners including but not limited to history/consultation, physical examination and other clinical skills in simulated clinical environments. They can also be used to give feedback and evaluate student performance.
}

Strongly Agree, Agree, Neutral, Disagree, and Strongly Disagree. The questions identified in this paper that concentrate on interpersonal skills include:

1. I think the feedback format for the clinical skills exam was effective.

2. The clinical skills feedback session will improve my communications with real patients.

The third question addressed the participants' overall experience with both the mannequin simulations and standardized patient encounters with feedback.

1.1.3. This experience has helped me to continue to develop the skills necessary to care for patients.

In addition, participants also had the opportunity to add their comments and or suggestions about the program on the survey.

Data Analysis: The results indicated that during the identified years (2011-2012, 2012-2013) participants overwhelmingly felt that the feedback portion of the M3 Come Home Day Curriculum was not only a positive experience for students but indicated that this program was valuable for future participants. $85 \%$ or greater of the medical students thought that the program was effective. (See the survey questions and graphs below).
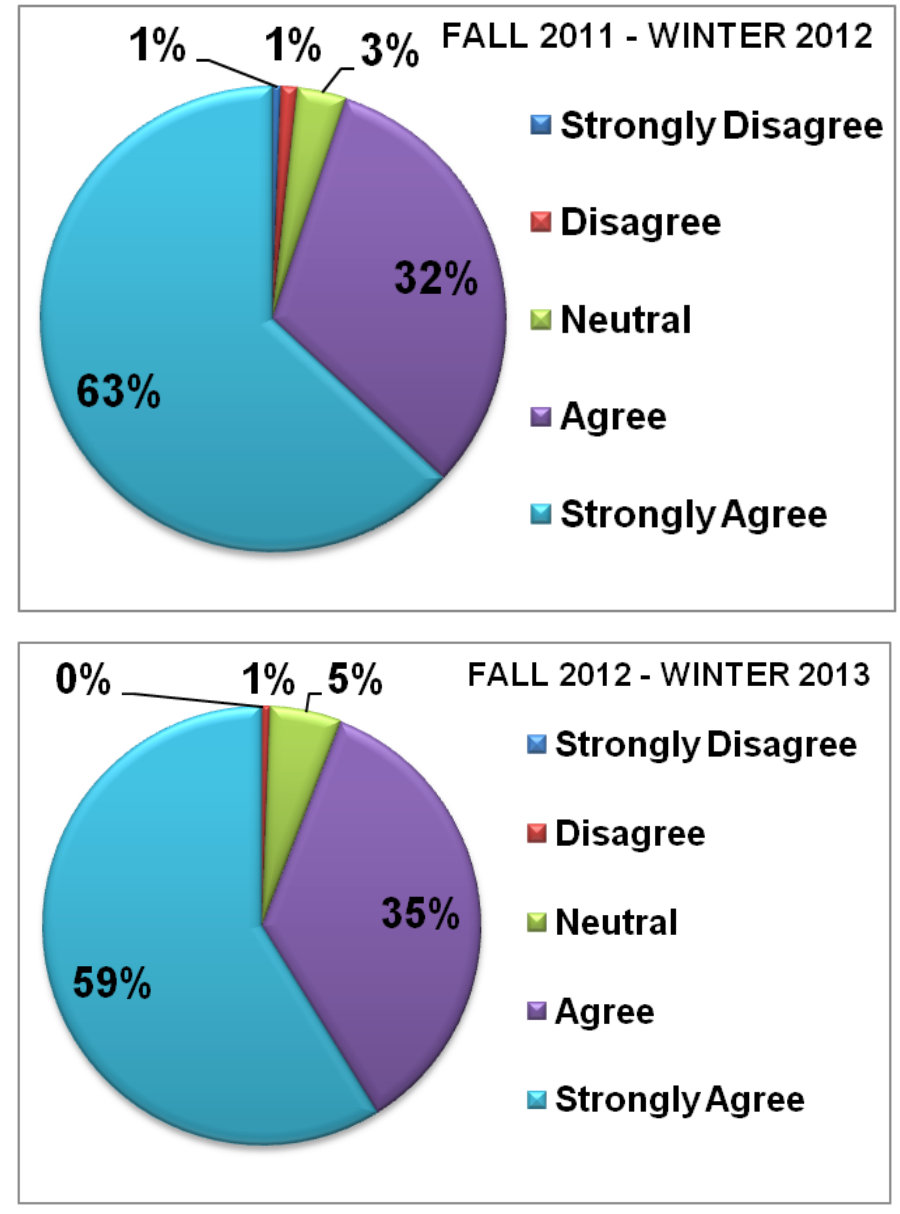

I think the feedback format for the clinical skills exam was effective 

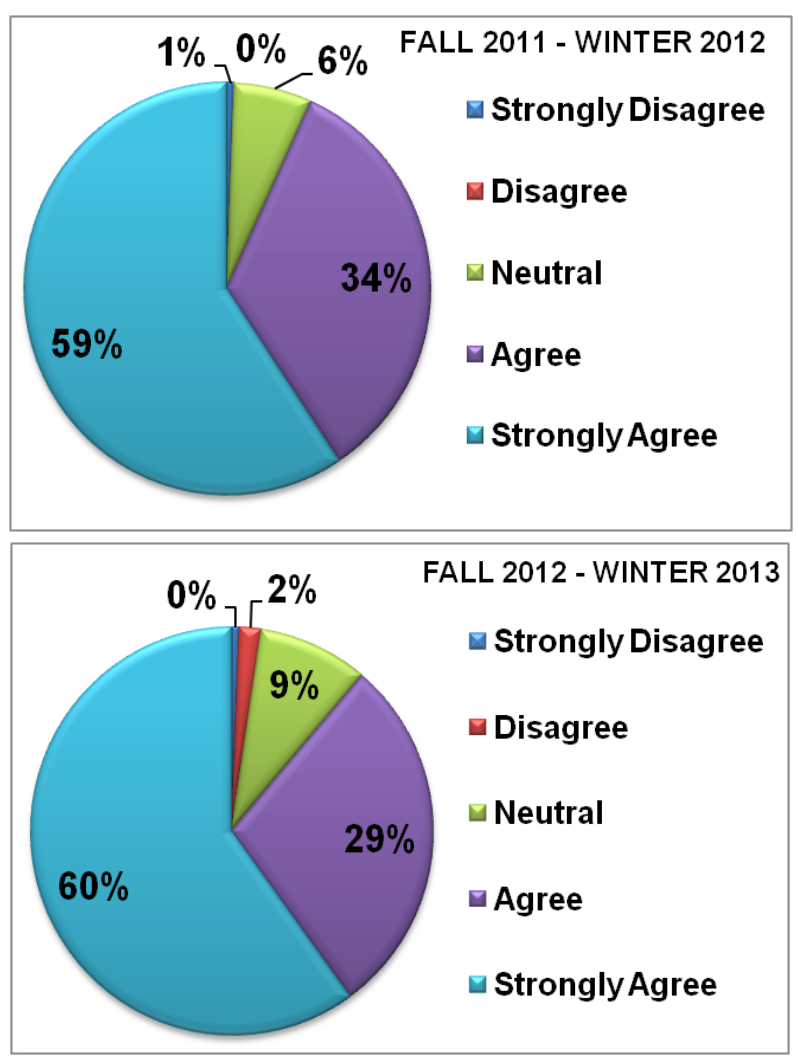

The clinical skills feedback session will improve my communication with real patients
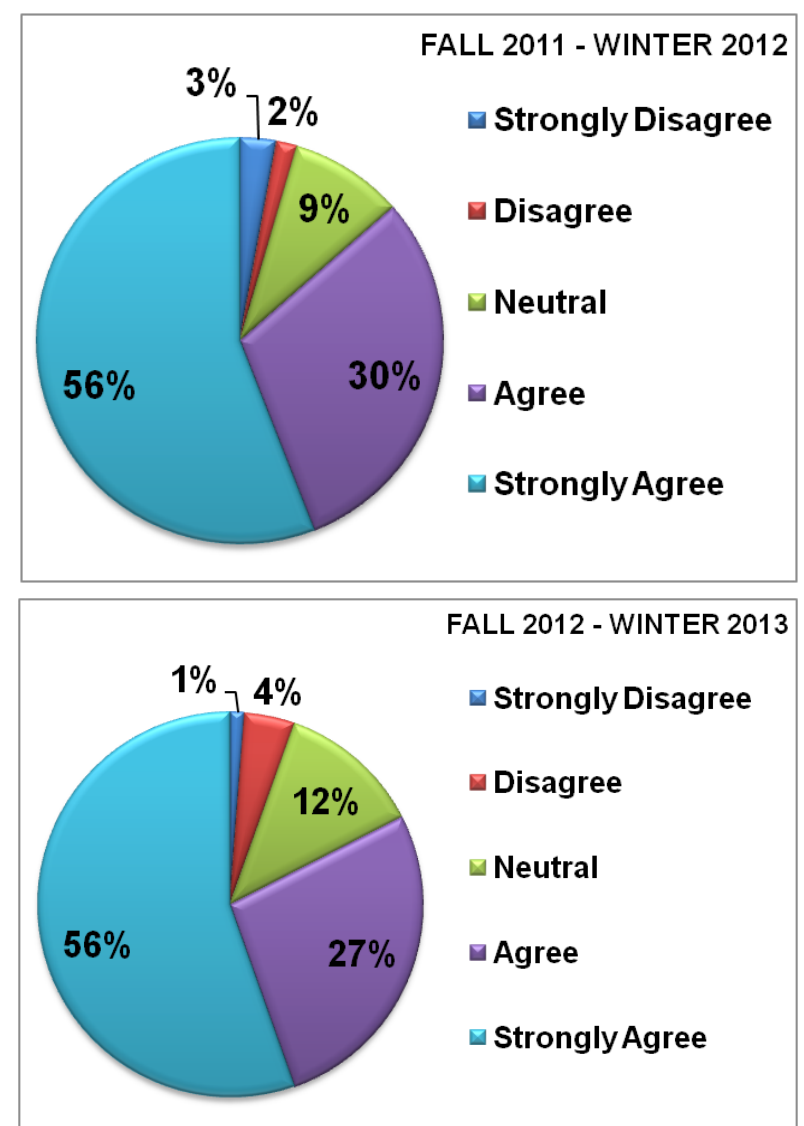

The clinical skills feedback session will improve my communication with real patients
Prominent Themes: We also identified prominent themes that occurred in the surveys that participants identified in the comment section of the survey. These comments culled from the data are revelatory $[1,2]$. Themes included: "exercise well spent", "opportunity to test critical thinking and decision-making skills", "great experience" "need to be implemented more often", and "watching the videos as a group was helpful". We feel that most importantly, participants identified that "patient simulations provided experiences that will help with real patients" as a true test of the humanity of this program.

\section{Discussion}

We realize that there is a variety of opinions as to medical student's experience with "standardized" vs. "real patient encounters", however, we believe that our program offered the chance for medical students to share their experiences in a valid and authentic manner with their peers and faculty members. We also believe that through this sharing of how they felt, and what they saw while watching not only their videos but the videos of their peers, they can identify both the strengths and weaknesses not only for the clinical aspects of the patient physician encounter, but the humanistic domain in communication, an area that is often overlooked. Fundamental in the practice of medicine is the need for physicians to develop and improve their interpersonal skills with patients. Osteopathic physicians in particular are committed to the practice of medicine using a mind, body, spiritual triumvirate and during medical school the opportunity to work in the simulation lab and interacting with simulated patients although, not always ideal, is a potent vehicle for modeling interpersonal skills to help elevate and improve the patient/physician encounter. Some medical students have difficulties in interpersonal skills and there can be an argument on both sides on whether these skills can be taught philosophically or not, however, this unique opportunity offers participants the chance to not only improve their interpersonal skills but to be cognizant of their strengths and weakness in a visual manner [2-4].

We use more difficult cases during this program to allow the student's humanistic side to be challenged. Some of the topics include erectile dysfunction, multiple sexual partners, fear of dying, and talking with adolescent patients. Also, we feel that the discussion is more in depth than with our first and second year video feedback sessions because our third-year students are out on clinical rotations rather than in the classroom. It makes the feedback sessions more interactive and authentic. We often hear student comments like, "I saw someone like this last week in the hospital and here is how I handled that similar situation". Or "I wish I said or did what you just did in the video when my patient on my rotation responded like that." Like most things in life when you can put it into practice soon after you learn a topic, the more likely you are to use the information [5].

\section{Conclusion}

Because the medical students receive feedback from faculty and peers, we believe that feedback from peers rather than professors is more substantive for some students than others. Future research will be structured around various questions including: How can faculty be exemplary models in the patient simulation lab? Can we teach interpersonal skills? What is the best way to teach interpersonal skills? When faculty model interpersonal skills are that the most effective manner of teaching? Is providing the opportunity to review one's video encounter with a simulated patient the best way to learn how to improve interpersonal skills? Does patient simulation provide for authentic experiences that can be later modeled by student physicians? We look forward to future research and debate. 


\section{References}

1. Berg K, Majdan J, Berg D, Veloski J, Hojat M (2011) A comparison of medical students self-reported empathy with simulated patients' assessment of the student's empathy. Jefferson Medical College of Thomas Jefferson University, US. Medical Teacher: 33: 389-391.

2. Bombeke K, Symons L, Debaene L, DeWinter B, Schol S, et al. (2010) Help, I'm losing patient centredness! Experiences of medical students and their teachers. Medical Education, Blackwell Publishing, Ltd.
3. Dikici MF, Yaris F (2007) Standardized and simulated patient programs in Ondokuz Mayis University School of Medicine: medical education. Turkiye Klinikleri Journal of Medical Sciences 27: 738-743.

4. Ishikawa K (2010) Can nonverbal communication skills be taught? Medical Teacher 32: $860-863$.

5. Peterson E, Porter M, Calhoun A (2012) A Simulation-Based Curriculum to Address Relational Crises in Medicine, Journal of Graduate Medical Education 4: 351356.

Copyright: (C2018 McCarthy H. This is an open-access article distributed under the terms of the Creative Commons Attribution License, which permits unrestricted use, distribution, and reproduction in any medium, provided the original author and source are credited. 